\title{
FRACTAL SELF-ORGANIZATION OF BACTERIA-INSPIRED AGENTS
}

\author{
YUFENG HUANG, IAN KRUMANOCKER \\ and MARC-OLIVIER COPPENS* \\ Howard P. Isermann Department of Chemical \\ and Biological Engineering, Rensselaer Polytechnic Institute \\ 110 8th Street, Troy NY 12180, USA \\ *coppens@rpi.edu \\ Received \\ Accepted \\ Published \\ In memoriam Benoit B. Mandelbrot, mentor and friend
}

\begin{abstract}
We develop an agent-based model as a preliminary theoretical basis to guide the synthesis of a new class of materials with dynamic properties similar to bacterial colonies. Each agent in the model is representative of an individual bacterium capable of: the uptake of chemicals (nutrients), which are metabolized; active movement (part viscous, part diffusive), consuming metabolic energy; and cellular division, when agents have doubled in size. The agents grow in number and self-organize into fractal structures, depending on the rules that define the actions of the agents and the parameter values. The environment of the agents includes chemicals responsible for their growth and is described by a diffusion-reaction equation with Michaelis-Menten kinetics. These rules are modeled mathematically by a set of equations with five dimensionless groups that are functions of physical parameters. Simulations are performed for different parameter values. The resulting structures are characterized by their fractal scaling regime, box-counting and mass-radius dimensions, and lacunarity. Each parameter influences the overall structure in a unique way, generating a wide spectrum of structures. For certain combinations of parameter values, the model converges to a steady state, with a finite population of agents that no longer divide.
\end{abstract}

Keywords: Fractal; Fractal Growth; Bacterial Colonies; Agent-Based Model; Dynamic Materials.

${ }^{*}$ Corresponding author. 


\section{INTRODUCTION}

The complexity of many fractal structures emerges from the iteration of simple rules. ${ }^{1,2}$ Interestingly, fractals share this feature with self-replicating machines and cellular automata, ${ }^{3,4}$ introduced by von Neumann, whom Benoit Mandelbrot credited in many ways as an important influence and advisor in early days. ${ }^{5}$ In such self-replicating machines, simple rules that govern the actions of individual mobile elements or "agents" may lead to fractal structures, with features that are invariant under magnification, over a wide range of scales.

Similarly, randomly moving bacteria on a Petri dish, partially starved from nutrients, assemble into complex organizations that are often fractal, sometimes reminiscent of DLA (diffusion limited aggregation). In DLA, ${ }^{6}$ a central seed grows into an approximately self-similar, fractal cluster when diffusing agents, following a random walk through space, hit and irreversibly attach to the growing cluster. However, bacteria are more complicated: they can divide (self-replicate); they absorb nutrients, which allows them to grow and change their internal state; they respond to their environment by reacting to chemical and mechanical actions, excreting chemicals themselves, to which other bacteria may react (e.g., quorum sensing). ${ }^{7}$ Real bacteria have complicated genetics and metabolism. They are not simple dots, but have a size, shape and content that is species dependent. They perform random walks, but not according to classical Brownian motion, because they are often self-propelled (using flagella), are able to respond to chemical gradients (via chemotaxis), and interact with the surrounding fluid. ${ }^{8}$

Despite these complications, models that abstract the actions and reactions of bacteria in a fashion similar to the DLA-model, using modified but still much-simplified rules compared to those of real bacteria, have been shown to produce patterns that closely resemble bacterial communities. $^{9-14}$ Also non-biological growth and aggregation phenomena occurring in colloidal systems, electrochemistry, dielectric breakdown, fluid flow in porous media (viscous fingering), etc... frequently produce fractal structures with surprisingly universal features, despite the differences in microscopic physics or physical chemistry. ${ }^{10,15-18}$ Nevertheless, the more complicated features of bacteria, including their ability to divide and act to their environment in different ways, leads to more complex dynamics and adaptability of a growing bacterial community, when compared to non-living systems.

A bacterial community, in many ways, behaves as a synergetic, multicellular organism. ${ }^{19,20}$ It is much more complex than the constituting microbes. A community could be manipulated in response to certain actions, be it genetic or environmental. While these features are very important for biology and biochemical engineering, ${ }^{21}$ this paper does not seek a more sophisticated model to describe the organization of bacterial communities. Instead, we explore the organization of agent-based systems that are bacteria-inspired.

The "agents" and their rules of action are based on the simplest, still physically and chemically meaningful representation of a complicated biological reality, in such a way that this representation might be applied to the design and synthesis of nonbiological elements or artificial cells. Like bacteria, these artificial cells develop into organizations. The question we raise is what organizations can be achieved based on these simple rules, with outcomes that depend, for each agent, on the immediate environment of the agent (e.g., the local concentration of nutrients), as is the case for a bacterium? Similar to bacteria, we call the organizations "colonies", and seek the structure of these colonies.

Our ultimate goal is to formulate nature-inspired design rules for new classes of adaptive materials, as part of our philosophy of nature-inspired chemical engineering (NICE). The NICE approach learns from the architecture and dynamics of natural systems with desirable properties, such as robustness, efficiency, scalability or adaptability, and then uses the underlying mechanisms that lead to these properties in the design and synthesis of innovative materials and reaction systems with properties similar to the natural systems. ${ }^{22-25}$

\section{AGENT-BASED SIMULATIONS}

\subsection{Overview}

Bacteria moving on a Petri dish may self-organize into fractal and other remarkable patterns, depending on the growth conditions. ${ }^{9,11-13}$ The goal of our simulations is to study the landscape of structures emerging from individual elements or "agents" with functions inspired by those of bacteria. Each agent performs a random walk on a two-dimensional 
surface. While an agent moves, it takes up nutrition from the environment, which it converts and uses to prolong its movement or to replicate. As we will see, this often results in fractal structures, like those observed in bacterial communities.

The state of each agent is characterized by a single variable: its mass, $m$, similar to the mass of a bacterial cell. Depending on the value of $m$, an agent can perform one of three actions. Below a certain threshold, $m_{\text {min }}$, an agent remains immotile, as it contains insufficient mass that can be converted into metabolic energy to propel itself through the viscous medium. Above this minimum, but below a maximum mass, $m_{\max }$, an agent is motile and propels itself through the environment. When the mass of an agent reaches $m_{\max }$, it self-replicates, splitting into two equally sized, separate agents. Each of these agents has half the mass of the original, hence $m_{\max }=2 m_{\min }$.

The environment in which the agents carry out these actions contains nutrition, which serves as a fuel to the individual agents. Hence, the twodimensional environment is represented by a layer in which nutrients diffuse and are taken up by the agents. When an agent absorbs nutrients from the layer, the concentration of nutrients in proximity to this agent decreases. Nutrients are metabolized and partially converted into biomass that is stored inside the agent. As nutrients are depleted, a concentration gradient develops, inducing the diffusion of nutrients from areas of high concentration to low concentration.

\subsection{Agents}

In the current model, all actions of an individual agent, $i$, are a function of its mass, $m_{i}$, only. This mass, $m_{i}$, is determined by the amount of nutrient that agent $i$ absorbs and the actions it performs. In turn, the mass of an individual agent determines the actions it will perform. Absorption replenishes nutrients and therefore increases $m_{i}$, while movement consumes energy, which decreases $m_{i}$.

The size of an agent depends on its mass. As hypothesized, the only variable characterizing each agent is its mass. This assumption implies that the density of an agent, $\rho$, is the same for all agents, and is independent of the agent's size. All agents are modeled as circular, two-dimensional disks with a mass-dependent radius $R\left(m_{i}\right)$. Since $\rho$ is constant, the surface area of each twodimensional agent, $S_{i}$, is proportional to its mass, $m_{i}: S_{i}=m_{i} / \rho=\pi\left[R\left(m_{i}\right)\right]^{2}$. The size of an agent determines its total rate of absorption of nutrients, and its velocity, $v\left(m_{i}\right)$.

The mass $m_{\text {min }}$ is the minimum allowed mass of an agent in the simulation. This represents the minimum amount of material an agent needs to sustain itself. When the mass of an agent drops to $m_{\text {min }}$, it is still able to absorb nutrients, should they become available by diffusion, but it is unable to move. Agents falling into this category are referred to as dormant, stationary or "immotile". The immotile agents have a radius $R_{\min }=R\left(m_{\min }\right)=$ $\sqrt{m_{\min }} /(\rho \pi)$.

When an agent $i$ has a mass $m_{i}$ between $m_{\text {min }}$ and $2 m_{\text {min }}$, it is able to move and is referred to as "motile". Each agent performs a random walk. It moves a short distance in a straight line, then changes direction randomly, moves another distance in a straight line, and so on. The environment is assumed to be viscous and Newtonian, and motion is slow enough to apply Stokes' law to the straight excursions:

$$
F_{d}=-4 \pi \mu R\left(m_{i}\right) v\left(m_{i}\right)
$$

where $F_{d}$ is the frictional or drag force acting on a disk-shaped agent $i, \mu$ is the viscosity of the medium, $R\left(m_{i}\right)$ is the radius of agent $i$, and $v\left(m_{i}\right)$ is its velocity. In this study we assume every agent to have the same, autonomous propulsion mechanism, which exerts a force $F_{p}$ on its surroundings. In bacteria this might be via flagella. Newton's third law dictates that $F_{p}+F_{d}=0$, barring external forces acting on the agent. In this study, we assume that the propulsion force is independent of mass, an assumption that can be modified, depending on the nature of the agents. Under this assumption, the magnitude of the drag force, $\left|F_{d}\right|$, is constant. We also assume that viscosity $\mu$ is uniform across the surface. The differential of the work done by the agents, moving an infinitesimal distance, $d s$, is derived as follows:

$$
d W=F_{p} d s=\left|F_{d}\right| d s \quad \text { or } \quad \frac{d W}{d t}=\left|F_{d}\right| v(m) .
$$

The equation of motion of an agent's random walk is written as follows:

$$
\frac{d \vec{r}_{i}}{d t}=v\left(m_{i}\right)[\vec{i} \cos \theta(t)+\vec{j} \sin \theta(t)] .
$$

Here, $\vec{r}_{i}$ is the position of agent $i$ at time $t, \vec{i}$ and $\vec{j}$ are unit vectors along the directions of two orthogonal axes of the two-dimensional surface, 
$\theta$ is the angle at time $t$, randomly changed after certain time intervals. The velocity of agent $i$ depends on its mass, $m_{i}$, which, itself, depends on time.

Finally, when the mass of an agent, $m_{i}=2 m_{\min }$, the agent divides. As division occurs, a new agent is added to the system, next to its parent. Due to this condition, no agent has a mass greater than $2 m_{\text {min }}$, because it will have divided before that. The mechanism of division is as follows:

$$
\left\{\begin{array}{l}
m_{i}^{+}=\frac{1}{2} m_{i}^{-} \\
m_{N+1}^{+}=\frac{1}{2} m_{i}^{-}
\end{array}, \quad m_{i}^{-} \geq 2 m_{\mathrm{min}} .\right.
$$

The subscripts denote the agents. $N$ is the total number of agents in the system prior to division; a new agent $N+1$ is created when $i$ divides. The superscripts "-" and "+" denote the agents before and after division, respectively.

The overall mass balance for each agent is thus determined by the three actions: motion, division, and absorption of nutrition.

Absorption of nutrient by the agents is modeled by Michaelis-Menten kinetics, similar to many bacterial cells. According to the Michaelis-Menten mechanism, the local rate of depletion of nutrients per unit area, $r_{n}$ (would be volume in three dimensions), at position $\vec{r}$ and time $t$, is:

$$
r_{n}=\frac{r_{\max } C_{n}(\vec{r}, t)}{K_{M}+C_{n}(\vec{r}, t)},
$$

where $r_{\max }$ is the maximum reaction rate, $C_{n}(\vec{r}, t)$ is the local concentration of nutrients at position $\vec{r}$ and time $t$, and $K_{M}$ is the Michaelis-Menten constant. This rate equation approximates first-order kinetics at low nutrition levels $\left(C_{n} \ll K_{M}\right)$ and zeroth-order kinetics at high nutrition levels $\left(C_{n} \gg K_{M}\right)$. Via metabolic processes, absorbed nutrients are converted into added agent mass. Let $p$ be the stoichiometric coefficient of the reaction of nutrients to agent mass (allowing for other excreted products, which do not affect the process), then $p r_{n}$ is the rate of increase of agent mass per unit area of an agent. The total rate of absorption by agent $i$ (change in mass per unit time) is obtained by integrating over the area, $S_{i}$, occupied by agent $i$, leading to $S_{i} p r_{n}$, when the rate of absorption is assumed constant over this area.

Therefore, when $m_{\text {min }}<m_{i}<2 m_{\text {min }}$, the change in mass of an agent over time is described by:

$$
\begin{aligned}
\frac{d m_{i}}{d t} & =p r_{n}\left[C_{n}(\vec{r}, t)\right] S_{i}\left(m_{i}\right)-\frac{1}{\Delta H} \frac{d W_{i}}{d t} \\
& \cong p r_{n}\left[C_{n}(\vec{r}, t)\right] \frac{m_{i}}{\rho}-\frac{\left|F_{d}\right| v_{i}\left(m_{i}\right)}{\Delta H}
\end{aligned}
$$

where Eq. (2) was used, the area occupied by an agent, $S_{i}=m_{i} / \rho$, and $\Delta H$ is the enthalpy change associated to the conversion of mass to metabolic energy.

\subsection{Nutrient Layer}

Locally, nutrients are consumed by the agents. This process results in a concentration gradient in the nutrition layer. Because nutrients diffuse from high to low concentration, the nutrition layer is described by the following diffusion-reaction equation for the nutrient concentration, $C_{n}$ :

$$
\begin{aligned}
\frac{\partial C_{n}(\vec{r}, t)}{\partial t}= & D_{C}\left(\frac{\partial^{2}}{\partial x^{2}}+\frac{\partial^{2}}{\partial y^{2}}\right) C_{n}(\vec{r}, t) \\
& -\delta(\vec{r}) r_{n}\left[C_{n}(\vec{r}, t)\right],
\end{aligned}
$$

where $r_{n}$ is the rate of depletion of the nutrients, as described by Eq. (5), and $\delta(\vec{r})$ represents the presence of agents at location $\vec{r}: \delta(\vec{r})=1$ if there is an agent $i$ with $m_{\min } \leq m_{i} \leq 2 m_{\min }$ at $\vec{r}$; otherwise, $\delta(\vec{r})=0$.

\section{MODEL IMPLEMENTATION AND ANALYSIS TOOLS}

\subsection{Dimensionless Formulation}

To study this agent-based model in a more general way, the equations are cast in a dimensionless form. Thus, the dimensionless mass $X_{i}(\vec{r}, \tau)$, dimensionless nutrient concentration $Y(\vec{r}, \tau)$, dimensionless time $\tau$, and dimensionless velocity $v^{\prime}$ are introduced, with the following relationships to the corresponding non-dimensionless physical variables and parameters:

$$
\begin{aligned}
m_{i} & =m_{\min }\left(1+X_{i}\right) & & 0 \leq X_{i}<1 \\
C_{n} & =C_{\max } Y & & 0 \leq Y \leq 1 \\
t & =\frac{R_{\min }}{v_{\max }} \tau & & 0<\tau \\
v_{i} & =v_{i}^{\prime} v_{\max } & & 0 \leq v_{i}^{\prime} .
\end{aligned}
$$


The maximum velocity of an agent corresponds to its minimum mass; from Eq. (1): $v_{\max }=\left|F_{d}\right| / 4 \pi \mu R_{\min }$. The dimensionless Laplacian operator is used, by normalizing the coordinates $x$ and $y$ to the minimum radius of the agents, which is the radius when the agents are immotile:

$$
\frac{\partial^{2}}{\partial x^{2}}+\frac{\partial^{2}}{\partial y^{2}}=\frac{1}{R_{\min }^{2}} \nabla^{2}
$$

Equation (7) for the diffusion of nutrients thus becomes, after substituting Eq. (5):

$$
\begin{cases}\frac{\partial Y}{\partial \tau}=D \nabla^{2} Y-B \frac{Y}{Y+A} & \text { in the presence of agent } i, \text { with } 0 \leq X_{i}<1 \\ \frac{\partial Y}{\partial \tau}=D \nabla^{2} Y & \text { in the absence of agents. }\end{cases}
$$

Depending on their state, each agent $i$ is described by the following equations, where Eqs. (14a) and (14b) are the dimensionless forms of Eqs. (4) and (6):

$$
\begin{cases}\frac{d X_{i}}{d \tau}=C \frac{Y}{Y+A}\left(1+X_{i}\right) & \text { for } X_{i}=0 \\ \frac{d X_{i}}{d \tau}=C \frac{Y}{Y+A}\left(1+X_{i}\right)-E \frac{1}{\sqrt{1+X_{i}}} & \text { for } 0<X_{i}<1 \quad i=1, \ldots, N \\ X_{i}^{+}=0 ; X_{N+1}=0 & \text { for } X_{i}^{-}=1 .\end{cases}
$$

The first equation, (14a), holds for immotile agents, which are still able to absorb nutrients (right-hand side term), resulting in accumulation of mass, but do not lose mass through motion, as in the last term of Eq. (14b). Here, the dimensionless velocity was used, which follows from Eqs. (1) and (8):

$$
v_{i}^{\prime}=\frac{1}{\sqrt{1+X_{i}}}, \quad i=1, \ldots, N .
$$

In these equations, dimensionless parameters $A-E$ were introduced. Table 1 shows how they depend on physical parameters.

\subsection{Implementation}

The nutrition layer is discretized into a grid with unit box width $a$. Simulations were carried out for different values of $a$ to ensure that the results are grid size independent. For the reported simulations, $a$ is chosen to be $10 R_{\min }$, a value that is large enough for the approximation in Eq. (6) to hold, and small enough for the value of $Y$ within a grid cell not to change appreciably. Time $\tau$ is discretized into small time steps $\Delta \tau$.

During each time step $\Delta \tau$, coarse-grained versions of Eqs. (13) and (14) are solved. The diffusion equation for the nutrients is discretized using the Crank-Nicolson method. The trajectories of the agents are also coarse-grained over time steps $\Delta \tau$. An agent changes direction after a certain amount of time that is modeled by a Poisson distribution, such that the same direction is maintained for an average duration of $\lambda \Delta \tau$, where $\lambda$ is the mean of the Poisson distribution. $\lambda=10$ for all simulations, because $\lambda$ can be neither too small nor too large for the assumptions behind the coarse-grained approximation to be valid. It was verified that changes of $\lambda$ around this average did not influence the results.

The initial conditions are set to: $N=50 ; X_{i}=0$ and $\left|\vec{r}_{i}\right| \leq 10 R_{\min }$ for $i=1,2, \ldots, N ; Y(\vec{r}, 0)=1$, that is, nutrients are initially homogeneously, uniformly distributed. The boundary of the nutrition layer is considered to be a rigid wall, that

Table 1 Expressions for the Parameters $A, B, C, D$, and $E$ in Eqs. (13) and (14), in Terms of Physical Variables.

\begin{tabular}{lccccc}
\hline Dimensionless Parameter & $\boldsymbol{A}$ & $\boldsymbol{B}$ & $\boldsymbol{C}$ & $\boldsymbol{D}$ & $\boldsymbol{E}$ \\
\hline Relation to physical variables & $\frac{K_{M}}{C_{\max }}$ & $\frac{r_{\max } R_{\min }}{C_{\max } v_{\max }}$ & $\frac{R_{\min } p r_{\max }}{v_{\max } \rho}$ & $\frac{D_{c}}{R_{\min } v_{\max }}$ & $\frac{\left|F_{d}\right| R_{\min }}{m_{\min } \Delta H}$ \\
\hline
\end{tabular}


is: $\nabla Y=\partial Y / \partial n=0$ at the boundary. A simulation is ended when $N \geq 30,000$ or after 300,000 time steps $\Delta \tau$, whichever occurs first. The stopping condition is chosen such that the average simulation time is less than two days.

All simulations were performed on a computer cluster comprised of eight nodes of dual 6-core $2.4 \mathrm{GHz}$ processors with $16 \mathrm{~Gb}$ RAM per node. The average time for a simulation to complete is less than 15 minutes.

\subsection{Box-Counting Dimension}

The standard procedure is used to estimate the boxcounting dimension of the colony formed by the agents. ${ }^{1}$ First, the colony is covered by a square grid containing unit boxes of width $R^{\prime}$ (in units of $\left.R_{\text {min }}\right)$. The number of boxes with at least one agent is counted and is recorded as $N_{B}\left(R^{\prime}\right)$. By changing the value of $R^{\prime}$, the corresponding $N_{B}\left(R^{\prime}\right)$ is obtained. The box-counting dimension $D_{B}$ is estimated from the absolute value of the slope of the linear region in a plot of $\log _{10}\left[N_{B}\left(R^{\prime}\right)\right]$ vs. $\log _{10}\left(R^{\prime}\right)$.

\subsection{Mass-Radius Dimension}

The mass dimension is estimated in two different ways. The first way involves counting the number of agents within concentric circles of radius $R^{\prime}$ (again in units of $R_{\text {min }}$ ) around a central point. This center is obtained by averaging the position of agents in the vertical and horizontal directions. Then, the number of agents within a circle of radius $R^{\prime}$ is counted, and recorded as $N_{M}\left(R^{\prime}\right)$, for different values of $R^{\prime}$. The mass-radius dimension is calculated from the linear region in the plot of $\log _{10}\left[N_{M}\left(R^{\prime}\right)\right]$ vs. $\log _{10}\left(R^{\prime}\right)$.

However, because the density of the colony is likely to be different at different radii, a coarsegrained mass-radius method is also employed. First, the colony is covered by a grid of small boxes. Similar to the box-counting method, each box in the grid has a binary value, depending on the presence of agents in the box. The number of occupied boxes within a circle of radius $R^{\prime}$ is counted, and recorded as $N_{M}^{\prime}\left(R^{\prime}\right)$. A plot of $\log _{10}\left[N_{M}^{\prime}\left(R^{\prime}\right)\right]$ vs. $\log _{10}\left(R^{\prime}\right)$ is then used for a second estimate of the mass dimension, $D_{M}$.

\subsection{Lacunarity}

Fractal dimension(s) give an impression of the space-filling capability of a set, but are insufficient to fully characterize it, just like a dimension of 2 for a Euclidean object does not tell us whether the object is a circular disk or a rectangle. A valuable complementary characteristic is lacunarity, which indicates how (non-)uniform the set is. Low lacunarity corresponds to a uniform, homogeneous distribution of holes (Latin lacunae). ${ }^{1}$ Fractals of the same Hausdorff dimension may differ considerably in their lacunarity. On occasion, different estimators for fractal dimension, such as box-counting schemes and the mass-radius relation already suggest this, since the mass dimension depends on the choice of a center around which the mass is measured as a function of the distance from the center.

A fast gliding box algorithm is used to obtain a measure for the lacunarity of the colony of agents. $^{26,27}$ First, the colony is coarse-grained by employing a grid of small box width, $R^{\prime \prime}=1$ (in units of $R_{\min }$ ). Unlike the box-counting method, the number of agents within each box is now counted.

Second, the standard gliding box algorithm is implemented to calculate the lacunarity of this coarse-grained colony. The width of the gliding box, $R^{\prime}$, is varied and chosen to be an integer multiple of $R^{\prime \prime}$. The box is first placed at the upper left corner of the coarse-graining grid and the number of agents within the box is counted as $S^{\prime}$. The box is then glided through the whole grid with displacement $R^{\prime \prime}$ in each step. The number of occurrences of a particular value of $S^{\prime}$ is counted and recorded as $N_{R^{\prime}}\left(S^{\prime}\right)$. This number is normalized by the total number of gliding boxes of length $R^{\prime}$, and the result is denoted as $Q_{R^{\prime}}\left(S^{\prime}\right)$. By choosing different integer multiples $R^{\prime}$ of the width of the coarsegraining boxes, $R^{\prime \prime}, Q$ is obtained as a function of $S^{\prime}$ and $R^{\prime}: Q=Q_{R^{\prime}}\left(S^{\prime}\right)=Q\left(S^{\prime}, R^{\prime}\right)$. The first and second moments of $Q$ over $S^{\prime}$ are calculated as $Z_{1}=\sum_{S^{\prime}} S^{\prime} Q\left(S^{\prime}, R^{\prime}\right)$ and $Z_{2}=\sum_{S^{\prime}} S^{\prime 2} Q\left(S^{\prime}, R^{\prime}\right)$, respectively. Gliding box lacunarity is defined as $L\left(R^{\prime}\right) \equiv Z_{2} / Z_{1}^{2}$. The lacunarity plot is a graphical representation of $\log _{10}\left[L\left(R^{\prime}\right)\right]$ vs. $\log _{10}\left(R^{\prime}\right)$.

\section{NUMERICAL RESULTS AND DISCUSSION}

When different values of the parameters $A, B, C$, $D$ and $E$ are substituted into Eqs. (13) and (14), the agents self-organize into colonies with different structures. In Sec. 4.1 the case in which $A=0.25$, $B=4, C=0.5, D=1$ and $E=0.1$ is discussed in detail. These values are chosen as the "base case", because, as we will see, they generate an aggregate 
similar to DLA, with features observed in real bacterial colonies on a Petri dish. Then, in Sec. 4.2, we will explore the large family of spatially structured patterns that is formed by changing one parameter at a time.

\subsection{Base Case}

\subsubsection{Visual exploration of the overall geometry}

Benoit Mandelbrot had a remarkably developed visual sense. He loved geometry and liked to first carefully look at shapes, before studying them numerically, using statistical and mathematical tools. Let us do this for the base case. Nine simulations with the same parameter values $(A=0.25$, $B=4, C=0.5, D=1$ and $E=0.1$ ) were performed. Figure 1 is a representative illustration of the structure formed by the agents, once they have grown to a community of 30,000 . The main panel in Fig. 1 shows the macroscopic spatial distribution of the agents. The black points correspond to immotile agents, with $X_{i}=0$, and the red dots correspond to motile or active agents, with $X_{i}>0$.

Globally, at the macro-scale, our eyes tell us that this colony is a self-similar fractal, reminiscent of DLA. ${ }^{6}$ Most active agents are close to the rim. Zooming in to an intermediate, meso-scale, as shown in two enlarged regions below and on the right of the main image, the branched structure is observed to contain numerous holes, not only in between, but also within the branches of the DLA-like cluster. These branches consist of clouds of agents over a width on the order of the nutrient grid size, $a \sim 10 R_{\text {min. }}$. Further zooming in, down to the micro-scale, where individual agents are clearly perceived, self-similarity vanishes. Agents are scattered randomly, but much more densely near the center of the cluster than near the active rim. The changes in meso- and microstructure over the cluster suggest multifractality.

\subsubsection{Fractal dimensions}

Since the colony formed by the growing agents contains self-similar features, fractal dimensions are calculated. First, by employing the box counting method, as show in Fig. 2a, the fractal dimension $D_{B} \approx 1.75$. The lower cutoff for the scaling regime occurs at $\log _{10}\left(1 / R^{\prime}\right)=-1$, or $R^{\prime}=10$. However, this scale corresponds to the width, $a$, of the boxes of the nutrition grid. It is obvious that this is the smallest scale at which we could obtain meaningful information about the structure.

Mass-radius dimensions are also calculated and compared with the box-counting dimension. As shown in Fig. 2b, the mass distribution along the radial direction is non-uniform: the dotted line shows two distinct regions that approximately follow power laws. One has an exponent of 2.19, while the other one has an exponent of 1.17. Both are very different from the box-counting dimension. The crossover occurs around $\log _{10}\left(R^{\prime}\right) \approx 1.2$. The inconsistency with the box-counting dimension is due to the fact that the local densities of the agents vary between regions that are at different distances from the center. Therefore, the mass-radius dimension is recalculated by coarse-graining the colony.

The colony is coarse-grained below a length scale $a=10 R_{\text {min }}$, because this is the scale below which nutrients are considered uniformly distributed. Also, this is the lower cutoff of the fractal regime measured by box counting. A log-log plot of the coarse-grained mass-radius relation is shown in Fig. 2b. The trend is linear with a slope $D_{M}^{\prime} \approx 1.87$, over the entire meaningful, coarse-grained range. Further coarse-graining $\left(20 R_{\min }, 30 R_{\text {min }}\right)$ has no effect, confirming the fractal, self-similar structure at scales above $a=10 R_{\min }$. The box-counting dimension of the coarse-grained object is still $D_{B} \approx$ 1.75 , because the boxes are wider than the inner cutoff of the fractal regime.

The difference between the coarse-grained mass dimension, $D_{M}^{\prime}$, and box-counting dimension, $D_{B}$, is due to the slow convergence of $D_{M}^{\prime}$. If simulations are carried out up to much larger colony sizes, say, $170,000, D_{B}$ remains almost the same, changing from 1.75 to 1.73 , while $D_{M}^{\prime}$ decreases significantly, from 1.87 to 1.79 . For very large clusters, it is indeed expected that $D_{M}^{\prime} \sim D_{B}$. However, simulation time increases from $1 / 2$ hour to 48 hours when simulating clusters with 170,000 agents instead of 30,000 in the base case. As, in our further analysis, we use the faster converging box-counting dimension, estimated over a broader scaling range, we were able to compare simulations for up to 30,000 agents without affecting our conclusions.

\subsubsection{Lacunarity}

The lacunarity of a DLA cluster (Fig. 3c) is calculated along with that of our colony of agents (Fig. 3b), because the colony displays similarities to DLA, including a similar fractal dimension. 


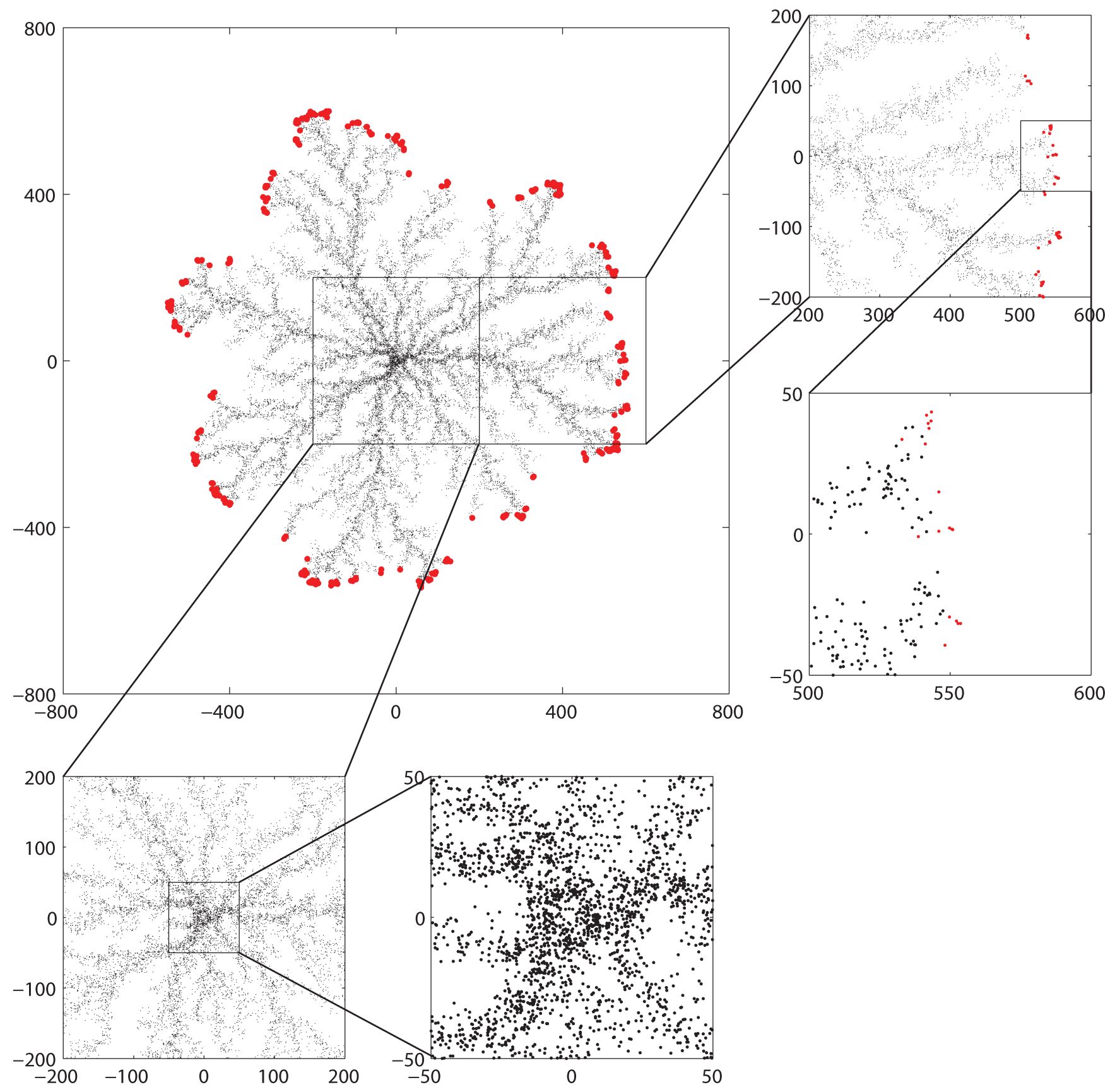

Fig. 1 Multi-scale structure of a colony formed by agents that propagate according to the "base case" scenario. Each agent is represented by an individual dot. The large central panel shows a macroscopic view of the entire colony. The figures on the right and bottom are enlarged regions of the colony, at two different levels of magnification, corresponding to a meso-scale (branches of finite width and variable density) and the micro-scale (individual branches). Red points represent active, motile agents $\left(X_{i}>0\right)$, while black points represent immotile agents $\left(X_{i}=0\right)$.

Figure 3a shows that the lacunarity curves of both structures almost coincide, but that the lacunarity of the colony is slightly larger across all scales. This means that the agents of the colony are less uniformly spaced, on average, than the elements in DLA. At first, this seems to be a surprising result, because the colony consists of scattered agents at the micro-scale (Fig. 1), unlike DLA, in which the individual elements are attached to one another. An explanation for this result is that the branches in the colony (meso-scale) are denser than the branches in DLA. When Figs. 3b and 3c are coarsegrained to the meso-scale (above scale $a$ ), DLA and the colony are almost indistinguishable. The 


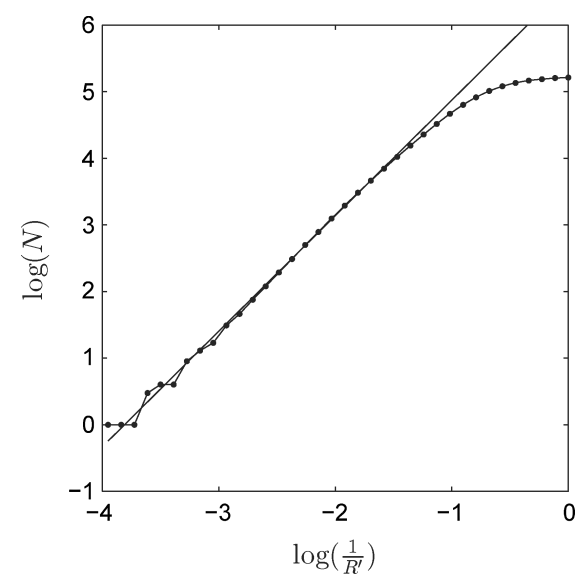

(a)

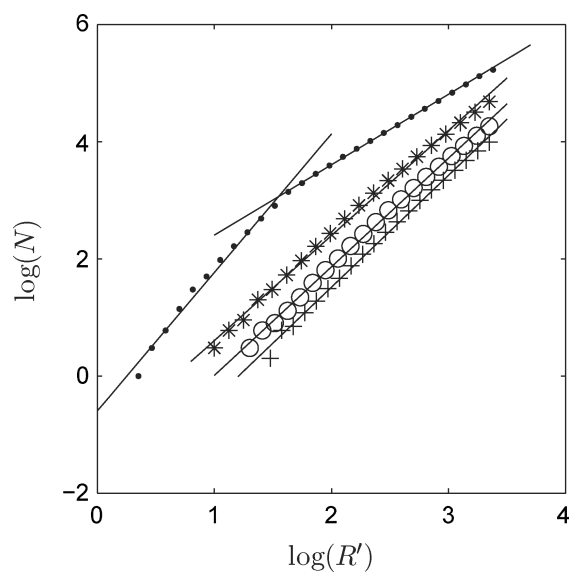

(b)

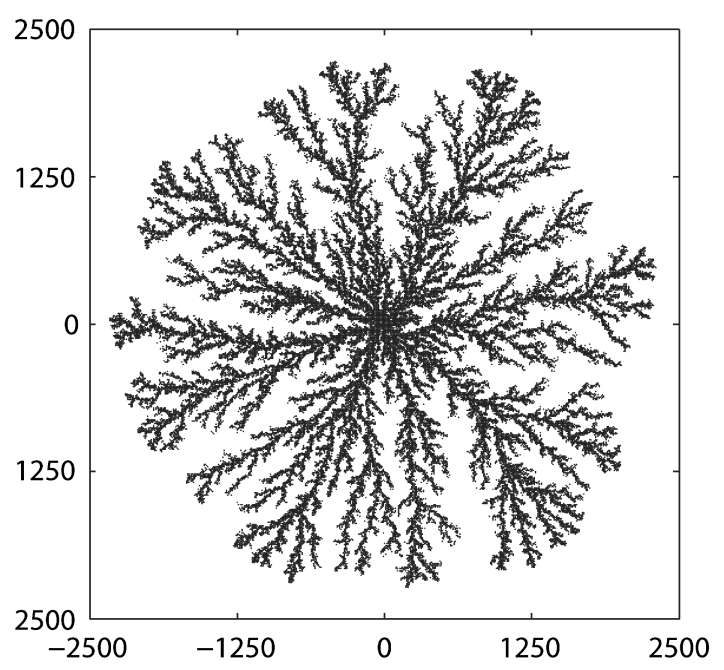

(c)

Fig. 2 Plots to estimate the fractal dimension of the colony in Fig. 1. The left graph is the box-counting plot with slope $D_{B} \approx 1.75$. The middle graph includes the standard mass dimension plot $(\cdots)$, and plots to estimate the mass dimension, $D_{M}^{\prime}$, after coarse-graining with box widths of $10 R_{\min }=a(* * *), 20 R_{\min }(\circ \circ \circ)$ and $30 R_{\min }(+++)$. The graph on the right shows the resulting colony (in units of $R_{\min }$ ) after coarse-graining below scales $10 R_{\min }=a$.

lacunarity plot provides quantitative information to both the similarity and the differences between DLA and the base case.

\subsubsection{Mass distribution of the agents}

The structure of the colony is a result of the active motions and divisions of the agents. The dynamics of the growing colony can be understood by examining the masses of the agents, because the actions performed are determined by their masses.

As shown in Fig. 1, the active agents spread sparsely at the tips of the growing branches. The number of active agents is small compared to the total population of the colony. Only $1.15 \pm 0.05 \%$ of the agents are active, but their role is crucial to the growth of the colony.

Each active agent behaves differently. As shown in Fig. 4, there is a mass distribution. The average mass of the active agents is $X \approx 0.5\left(m=1.5 m_{\text {min }}\right)$, which also happens to be a peak in the distribution. This is due to the special conditions at both ends of the mass distribution. At $X=0\left(m=m_{\min }\right)$, agents are no longer able to move, because their mass, and associated metabolism, is insufficient to generate kinetic energy. If the agents are also in the 


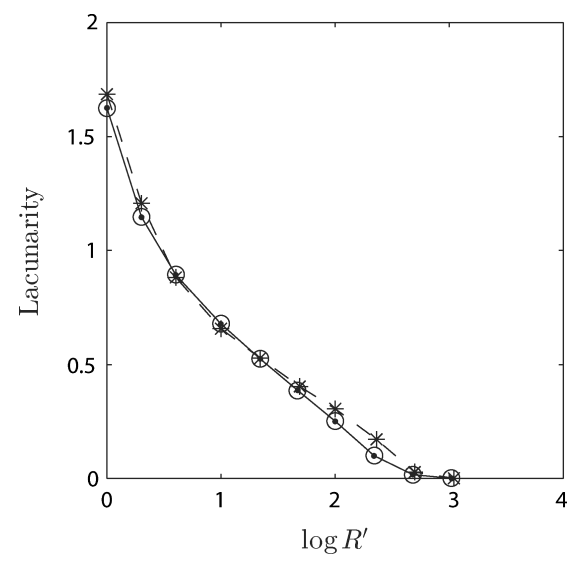

(a)

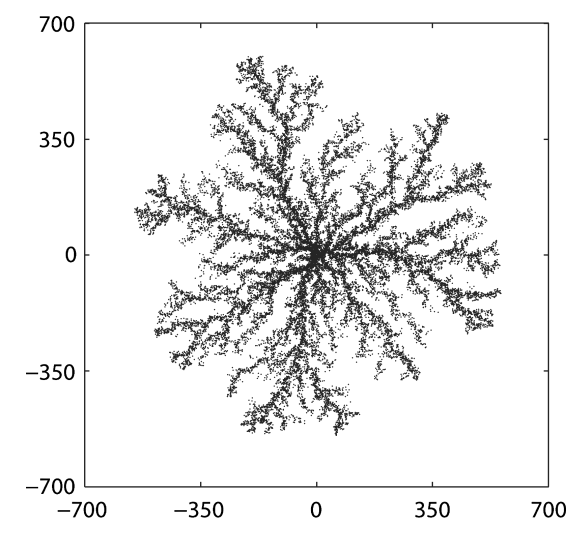

(b)

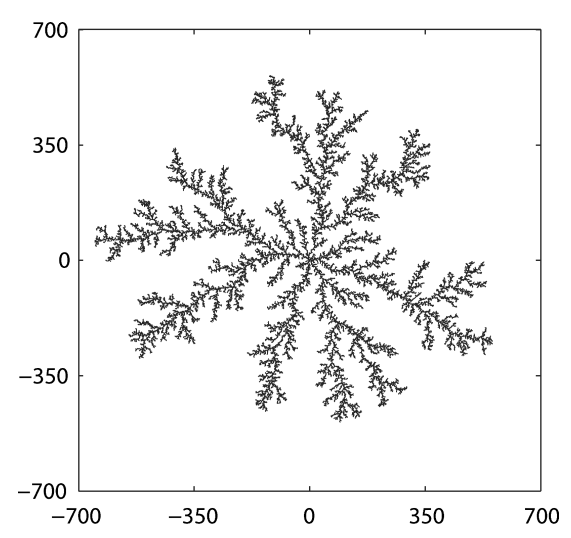

(c)

Fig. 3 Lacunarity plot (a) and comparison of the base case colony (b) and a DLA cluster (c), consisting of 30,000 agents each. (a) In the lacunarity plot on the left, $(* * *)$ or — represents the lacunarity of the colony, and ( $\circ \circ \circ)$ or - represents the lacunarity of the DLA cluster. (c) The DLA cluster on the right is drawn at the same scale as the colony in the middle graph, (b).

region less accessible to nutrition, it is very likely that the agents will stay immotile indefinitely. At $X=1\left(m=2 m_{\min }\right)$, agents divide, so that the number of agents of this mass is always 0 , while an active agent that has just grown to a mass $X=1$ divides into active agents of mass $X=0$, which is one reason for the peak near $X=0^{+}$. The combination of the conditions at $X=0$ and $X=1$ leads to an average mass of $X=0.5$ when a dynamic equilibrium has been reached. Scatter in the distribution is due to the small number of active agents $(345 \pm 14)$.

The peak at $X=0^{+}$corresponds to starving agents, entering the immotile state, or agents resulting from division. It is also possible that some inactive agents become active again by absorbing a minor amount of nutrition that reaches them via diffusion. $X=0^{-}$is the highest peak, by far, and is not shown in the figure, because it is three orders of magnitude higher. It corresponds to inactive agents, which contribute to the main, quasi-static structure of the colony, because they are stationed at certain locations.

\subsection{Parametric Study}

The colony discussed in the previous section is just one example of a large family of organizations generated by the agent-based model. By changing the values of the parameters, other organizations emerge. In order to understand the effects of each parameter, one parameter is changed at a time, while the values of the other parameters are kept the same as in the base case. 


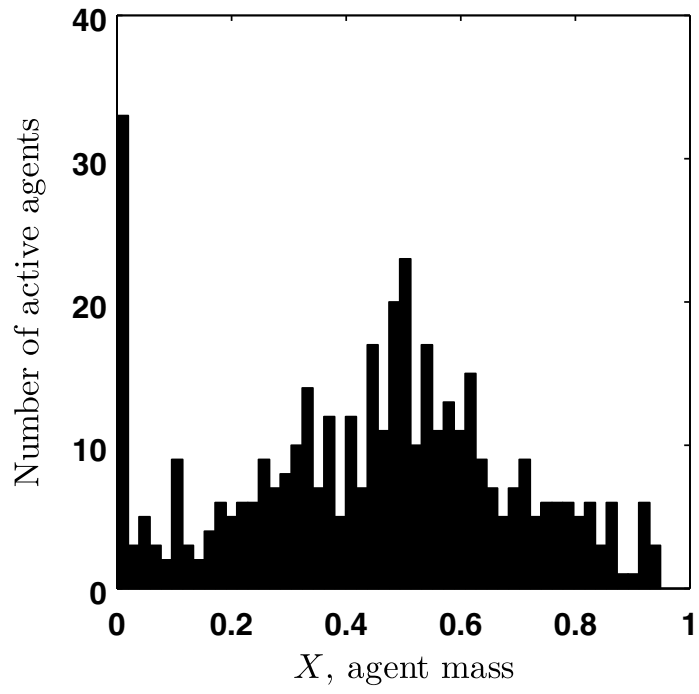

Fig. 4 Absolute frequencies of the dimensionless mass, $X$, of the active, motile agents in the base-case simulation. Most agents are immotile, with $X=0^{-}$.

\subsection{1. $\quad$ Effect of kinetics $(A)$}

$A$ is the most influential parameter in the model, because it is directly related to the kinetics of absorption of nutrients by the agents. Mathematically, this parameter links the Eq. (13) for the nutrition layer to those for the system of agents, Eq. (14). Therefore, the structure of the colony is strongly dependent on $A=K_{M} / C_{\max }$, which is the Michaelis-Menten constant made dimensionless by division by the maximum (initial) concentration of nutrients. As $A$ approaches 0, the absorption kinetics, which are proportional to $Y /(Y+$ $A)$, can be approximated as zeroth-order (constant). On the other hand, when $A$ is large $(A \gg$ $Y)$, the absorption of nutrition can be approximated as first-order with reduced rate constant $(\sim Y / A)$.

Numerical simulations were performed for $A=0$ to 4 , with increments of 0.4 . The structure of the colony strongly depends on $A$. Figure $5 \mathrm{a}$ is a plot of the box-counting dimension, and Fig. 5b shows the ratio of active agents to non-active agents, both as a function of $A$. The graphs show similarities, namely: when $A$ is close to 0 , the slope is large and positive; when $A$ is high, the slope is large and negative; and when $A$ has an intermediate value, the slope is small and negative. It is obvious that these three regions correspond to different types of organization, as shown in Fig. 5c.

The first region, where $A \rightarrow 0$, represents organizations with zeroth-order absorption kinetics. Local
Fractal Self-Organization of Bacteria-Inspired Agents

11

nutrition concentration is depleted to 0 at its maximum rate, according to Eq. (5), since it is linear in time and does not depend on its current level. As a result, nutrition from outside of the colony is not able to diffuse to the inside of the colony, because it is quickly absorbed by the agents at the perimeter. The number of active agents is thus decreasing as $A \rightarrow 0$, as shown in Fig. 5b. Due to the fast depletion rate of nutrients, agents moving in a radial direction are favored because it allows them to grow further. This selection of a particular direction is responsible for the thin branches shown in the first colony in Fig. 5c, which reminds us of the lightning-like, fractal patterns formed by the dielectric breakdown model (DBM). ${ }^{10}$ As expected, the box-counting dimension of these colonies is low, as shown in Fig. 5a.

The other extreme, for which $A \gg 1 \geq Y$, corresponds to first-order absorption kinetics. Contrarily to the high absorption rate in the zeroth-order reaction, nutrition is not depleted as quickly. However, despite the presence of nutrients, the number of active agents does not keep increasing. This is because agents consume their mass to generate kinetic energy, and they shrink when the absorption rate is so low that it does not counter-balance the required metabolic energy. Hence, the mass of each agent tends to its minimum $(X=0)$, and the agent becomes immotile. The result is a non-fractal colony with few branches. A typical example, for $A=4$, is shown in the rightmost panel of Fig. 5c. A boxcounting dimension slightly above 1 is obtained, but this non-integer value is biased by the small size of the colony.

For intermediate values of $A$, the agents selforganize into colonies with a fractal structure. A sharp maximum was obtained around the base case ( $A=0.25)$, for which the box-counting dimension of 1.75 is close to that of DLA. Colonies for higher values of $A$, but below 4, also have a self-similar structure of gradually decreasing box-counting dimension. Interestingly, these organizations keep growing up to and beyond the maximum simulated size of 30,000 agents. For the base case, a simulation up to 170,000 agents did not reveal differences in the general structure of the colony.

The presence of three regimes demonstrates how strongly the self-organization depends on the kinetics of absorption (0th order, Michaelis-Menten, 1st order kinetics), which is governed by the value of $A$. Interestingly, the transition between these "phases" 


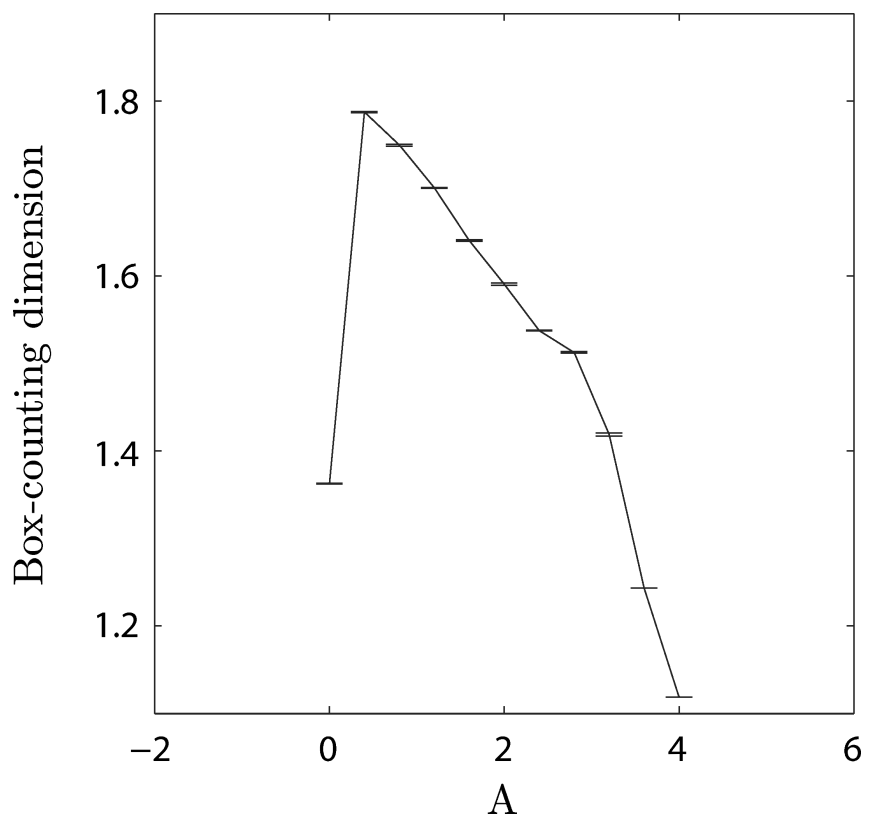

(a)

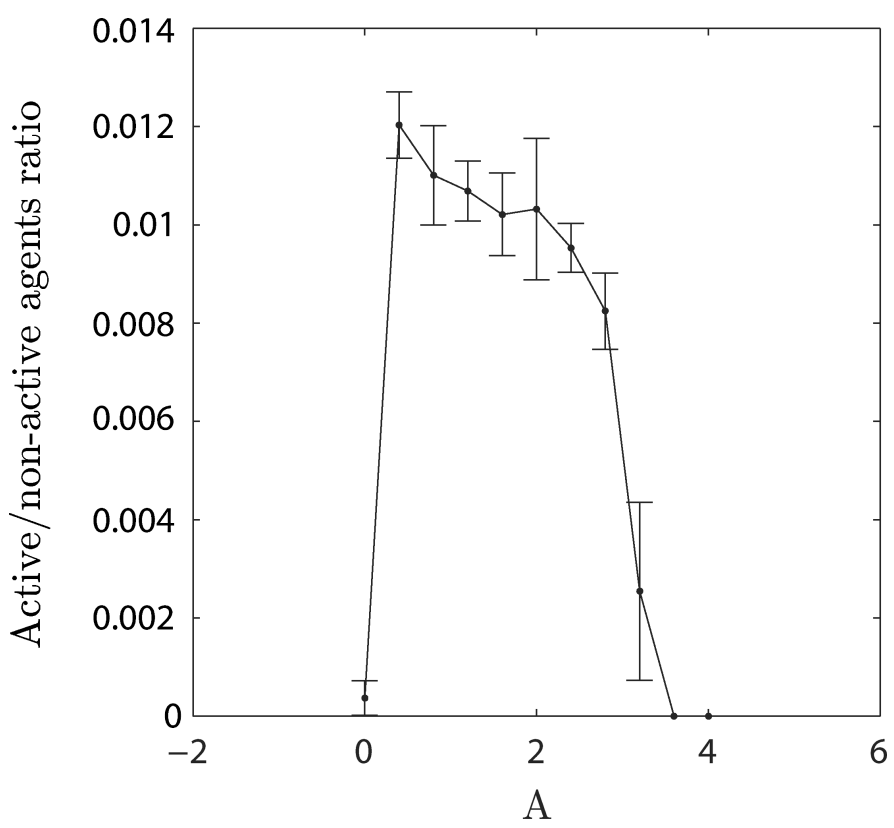

(b)

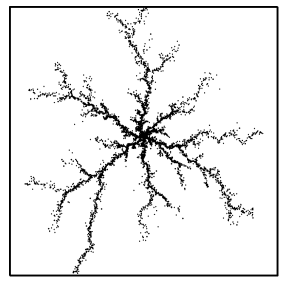

$A=0$

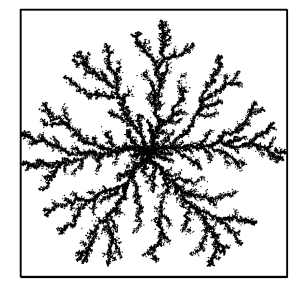

$A=1.6$

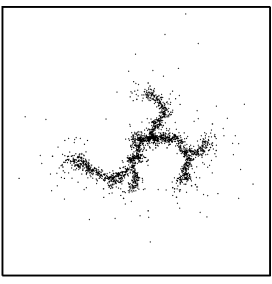

$A=4$

(c)

Fig. 5 Effects of $A$ on the colony. (a) Relationship between the box-counting dimension of the colony and the parameter $A$. (b) Relationship between the ratio of the number of active agents to the number of non-active agents, and the parameter $A$. (c) Structures of colonies for $A=0, A=1.6$ and $A=4$.

is due to the fact that the colonies reach a steady state, consisting of a finite number of agents, below and above critical values of $A$. We do not give explicit values for these critical thresholds, as longer simulations will need to be carried out to determine them with sufficient precision, but Fig. $5 \mathrm{~b}$ gives a good qualitative indication.

The general dynamics of the agent-based system are determined by the absorption of nutrition, which allows individual agents to move and divide, expanding the colony. However, these two actions - motion and division - have opposite effects on the total mass of the colony, because absorption increases mass, while motion consumes metabolic energy, hence decreases mass. When the colony reaches a certain size, the effects of the two actions cancel each other, leading to a steady state.
This happens both for 0th and 1st order kinetics, so that the colony reaches a finite size in both situations, even though the steady-state morphology of the system is very different. For example, the number of agents for $A=0$ stops increasing when it reaches $7200 \pm 190$. It is expected that the intermediate regime in Fig. 5 becomes narrower when the simulations are carried out beyond an allowed maximum of 30,000 agents, because some colonies for intermediate values of $A$ begin to reach a steady state. At this stage, it cannot be stated for certain whether the colony will be able to maintain indefinite growth within a certain range of values of $A$. For nutrient layers that are not infinitely extended, the boundary conditions for the nutrients (Dirichlet or Neumann) could play a role as well. 


\subsection{2. $\quad$ Effects of parameters related to agent motion and the consumption of nutrients $(B, C$ and $E)$}

Parameter $C$ is the constant pre-factor of the nutrient absorption term for the agents, in Eqs. (14a) and (14b). When $C$ is large, agents grow rapidly, and so does the colony. This is confirmed by the positive, linear relationship of the number of active agents with $C$ in Fig. 6d. The fractal dimension does not increase indefinitely, and seems to reach a plateau at higher values of $C$, even though the structures, shown at the bottom of Fig. 6, look different. The lacunarity (not shown here) decreases, as the structures become more uniform at the meso-scale. This is due to the rapid division at high values of $C$, leading to dense structures similar to those formed by the Eden process. ${ }^{10}$
Parameter $B$ is the constant pre-factor of the depletion term for the nutrition layer. The ratio $C / B=p C_{\max } / \rho$ is proportional to the initial concentration of nutrients. For the same concentration of nutrients, $C_{\max }$, a low value of $B$ and a high value of $C$ have similar effects. This explains why Figs. 6a-6c look like mirror images of each other, with respect to the base case in which $B=4$ and $C=0.5$. For the same value of $C$, a lower value of $B$ depletes the nutrients less rapidly, so that low-lacunar structures of high box-counting dimension appear (see Fig. 6a, and colony for $B=2)$. The fraction of active, motile agents is inversely related to $B$ for the same reason (Fig. 6b).

In Eq. (14b), parameter $E=\left|F_{d}\right| R_{\text {min }} /$ $\left(\Delta H m_{\text {min }}\right)$ determines the contribution of the mass $X_{i}$ that is expended to kinetic energy, accounting
B
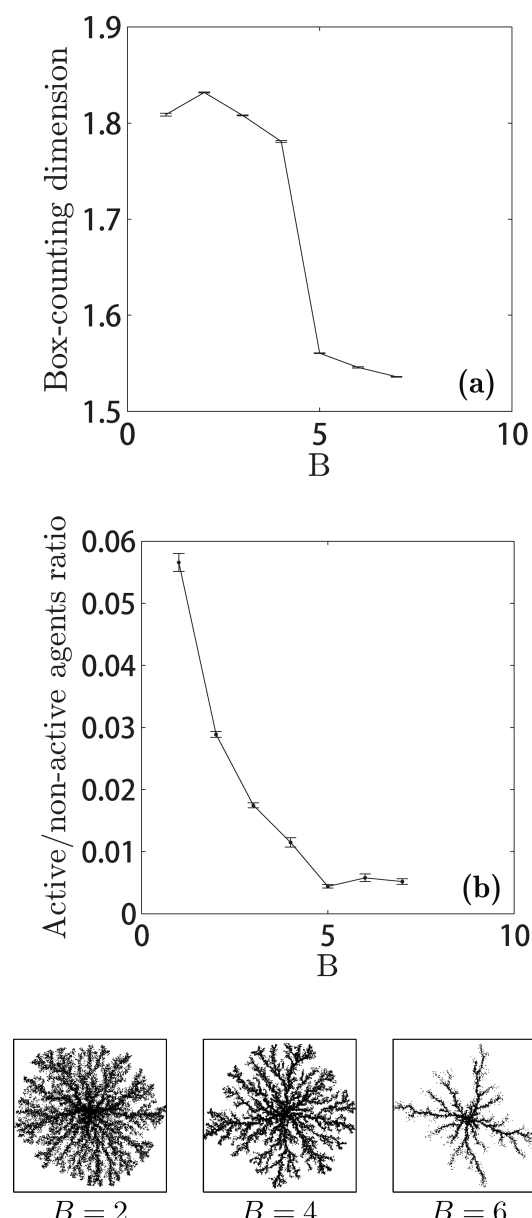

$B=4$

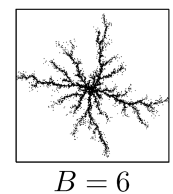

C
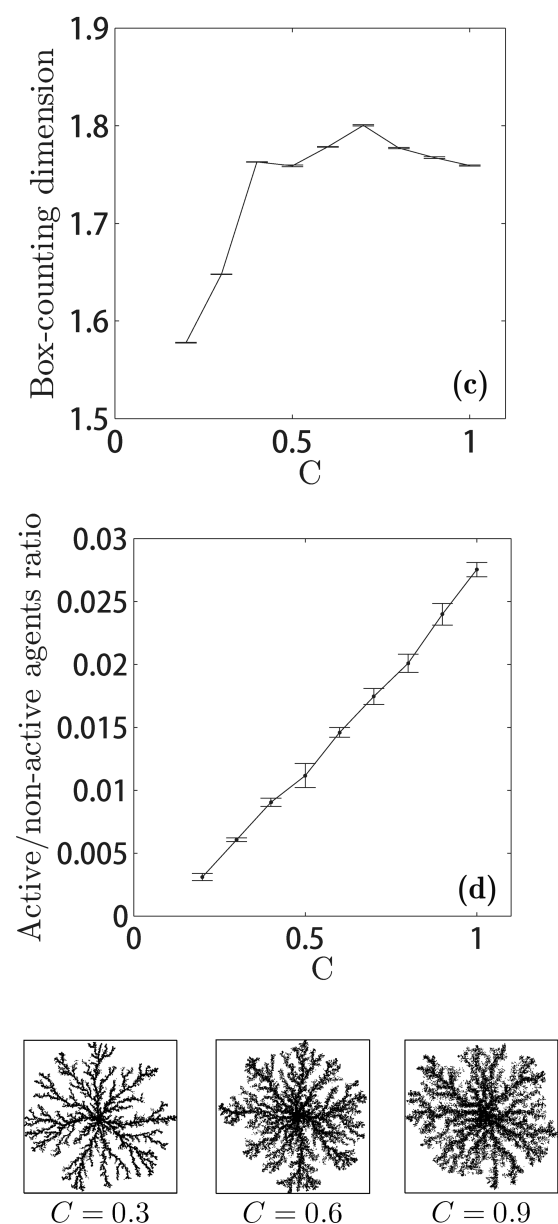
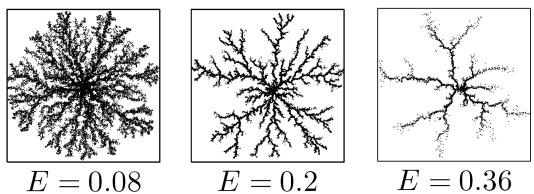

Fig. 6 The effects of parameters $B, C$, and $E$ to the structure of the colony. Top row: box-counting dimensions; below: ratio of active/non-active agents as a function of each parameter. Bottom: a few representative examples. 
for metabolic conversion (enthalpy change $\Delta H$ ). Note that $E=0.1$ corresponds to the base case. $E$ is the pre-factor of the negative term in Eq. (14b), while $C$ is the pre-factor of the positive term. Therefore, it is logical that both the fraction of active agents and the fractal box-counting dimension decrease with $E$. High values of $E$ lead to a Lichtenberg-like (DBM) pattern. At high values of $E$ the number of active agents tends to zero, so that a steady-state population is reached; $E$ is therefore another important parameter to control the system properties. Furthermore, Fig. 6e shows that the fractal dimension is approximately linear in $E$ over the investigated range. This is a useful feature when nature-inspired systems are designed.

\subsubsection{Effect of the diffusivity of the nutrients $(D)$}

Parameter $D$ is the dimensionless diffusivity of nutrients in the nutrition layer. It only appears in Eq. (13). The structures obtain their characteristic shapes mainly due to the limiting diffusion of nutrients to the agents, whose actions depend entirely on how much nutrition they are able to absorb. Parameter $D$ is, therefore, the main determinant of the branching process. Note that the scale in Fig. 7a is logarithmic. The base case corresponds to $\log (D)=0$. A family of branching patterns is formed within a quite narrow range of $D$. When $D$ is very large $(D>10, \log (D)>1)$, agents have easy

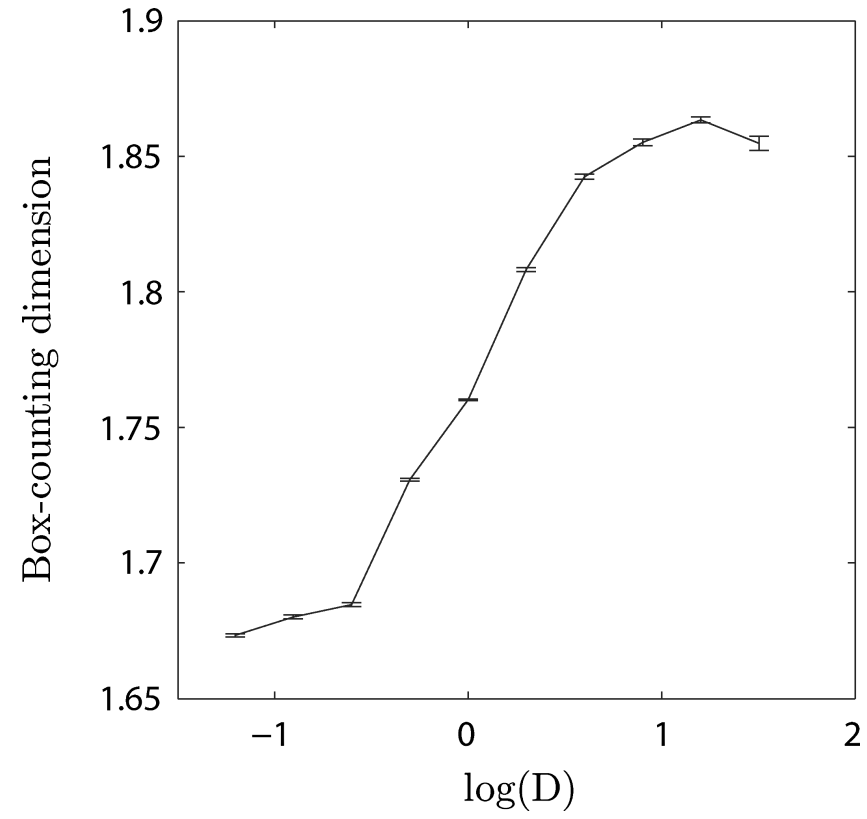

(a)

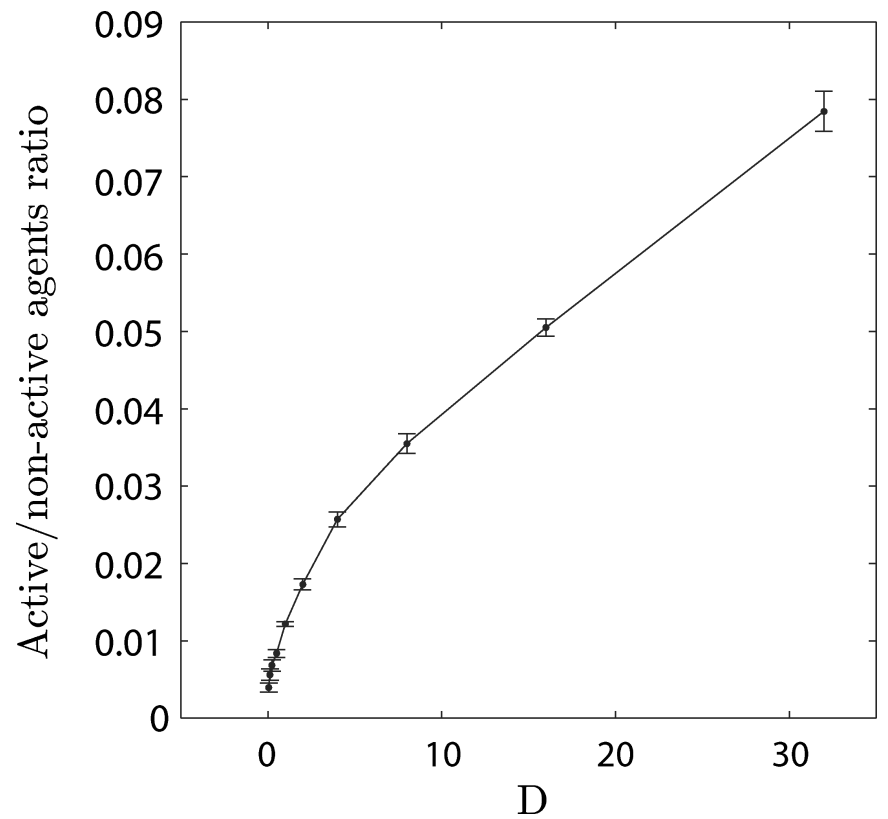

(b)

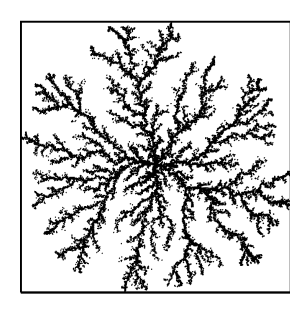

$D=0.25$

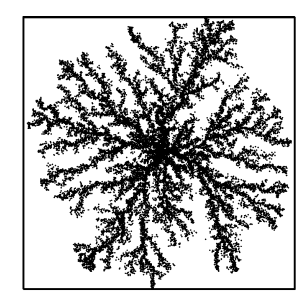

$D=1$

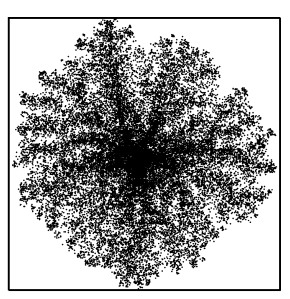

$D=16$

(c)

Fig. 7 Effect of dimensionless diffusivity of the nutrients, $D$, on the structure of the colony. (a) Top left: Box-counting dimension vs. $\log _{10}(D)$. (b) Top right: Ratio of active/non-active agents as a function of $D$. 
access to nutrition, which leads to similar effects as a high value of parameter $C$, but even more pronounced: the box-counting dimension saturates to a high value, as the colony becomes close to spacefilling, and a linear relationship between the number of active agents and $D$ is observed (Fig. 7b). At low values of $D$, the organization is DLA-like.

\section{CONCLUSIONS AND OUTLOOK}

Inspired by the adaptive behavior of bacterial colonies, we wish to design adaptive materials sharing similar properties. This study serves as a theoretical, exploratory basis for future experiments to develop these types of materials. Already, the simple agent-nutrient model in this study has been able to generate a variety of fractal and nonfractal structures of widely different geometry. The parametric study will be extended to include the simultaneous variation of multiple parameters. Multifractal formalisms, a deeper study of lacunarity, and a characterization of the dynamics should provide more detailed information beyond what is discussed in this introductory paper.

Although this agent-based system shows similarities to models that have been used to describe bacterial colonies, it is essentially different. First, this model could be used to design both biotic and nonbiotic systems, while the bacterial colony models are targeted at bacterial systems, which they aim to represent. Second, the model is based on physically realizable assumptions in non-biological systems. It is aimed less at being a reductionist approach for a biological system (for which it would be overly simplistic), than at offering a nature-inspired design methodology for non-biological systems (for which it contains as simple as possible ingredients to produce highly complex dynamics and morphologies).

The diffusion-reaction equations and the equations of motion are derived according to realistic physical laws. On the contrary, many bacterial colony models in the literature are based on assumptions unique to bacterial colonies. Moreover, in many of the bacterial colony models, the active and non-active states are defined as an assumption to the model to generate the desired branching structures. In our model, the active (motile) and non-active (immotile) states are the consequence of a mass-energy balance. Agents can be non-active at one moment and active at another moment, as long as nutrition is supplied.
Another remarkable result is that many of the systems generated from the presented model reach a dynamic steady state with a finite, limit number of agents. The values of the critical thresholds of the parameters, in terms of whether a system reaches such a dynamic steady state, should be evaluated as a function of system size and simulation time. The ability to control growth up to a certain size is useful for the synthesis of real materials: as long as the initial condition is set, the system is guaranteed to aggregate into a structure of a certain morphology when the steady state is reached. It would also be interesting to explore the consequences for biological systems, e.g., tumor growth.

The agent-nutrition model discussed in this study is simple, but easily extended to include the effects of quorum sensing, additional transmitters and receptors, as well as different agent dynamics or kinetics. Some of these extensions will be considered in future work, especially when they allow us to realize qualitatively different behavior. Even so, we strive for the simplest nature-inspired representation that could be realistically applied to design materials or systems with a desired, adaptive collective response by means of self-replicating agents.

\section{REFERENCES}

1. B. B. Mandelbrot, The Fractal Geometry of Nature (Freeman, San Francisco, 1983).

2. B. B. Mandelbrot, Fractals and Chaos: The Mandelbrot Set and Beyond (Springer, New York, 2004).

3. J. von Neumann, The general and logical theory of automata, in: Collected Works, ed. A. H. Taub, Vol. 5 (Pergamon Press, UK, 1961), pp. 288-326.

4. J. von Neumann and A. W. Burks, Theory of SelfReproducing Automata (University of Illinois Press, Urbana, 1966).

5. B. B. Mandelbrot, Private communication, see also http://www.webofstories.com (2011).

6. T. A. Witten and L. M. Sander, Diffusion-limited aggregation: A kinetic critical phenomenon, Phys. Rev. Lett. 47 (1981) 1400-1403.

7. B. L. Bassler, How bacteria talk to each other: Regulation of gene expression by quorum sensing, Curr. Op. Microbiol. 2 (1999) 582-587.

8. Y. Bozorgi and P. T. Underhill, Effect of viscoelasticity on the collective behavior of swimming microorganisms, Phys. Rev. E 84(6) (2011) 061901.

9. M. Ohgiwari, M. Matsushita and T. Matsuyama, Morphological changes in growth phenomena of bacterial colony patterns, J. Phys. Soc. Japan 61 (1992) $816-822$. 
10. T. Vicsek, Fractal Growth Phenomena, 2nd Edition (World Scientific, Singapore, 1992).

11. T. Matsuyama, R. M. Harshey and M. Matsushita, Self-similar colony morphogenesis by bacteria as the experimental model of fractal growth by a cell population, Fractals 1(3) (1993) 302-311.

12. E. Ben-Jacob, O. Schochet, A. Tenenbaum, I. Cohen, A. Czirók and T. Vicsek, Communication, regulation and control during complex patterning of bacterial colonies, Fractals 2(1) (1994) 15-44.

13. M. Matsushita, J. Wakita, H. Itoh, I. Rafols, T. Matsuyama, H. Sakaguchi and M. Mimura, Interface growth and pattern formation in bacterial colonies, Physica A 249 (1998) 517-524.

14. C. I. Golding, Y. Kozlovsky and E. Ben-Jacob, Continuous and discrete models of cooperation in complex bacterial colonies, Fractals 7(3) (1999) 235-247.

15. M. Kolb, R. Botet and R. Jullien, Scaling of kinetically growing clusters, Phys. Rev. Lett. 51 (1983) 1123-1126.

16. D. A. Kessler, J. Koplik and H. Levine, Pattern selection in fingered growth phenomena, Adv. Phys. 37 (1988) 255.

17. E. Ben-Jacob and P. Garik, The formation of patterns in non-equilibrium growth, Nature 343 (1990) $523-530$.

18. F. Family and T. Vicsek (eds.), Dynamics of Fractal Surfaces (World Scientific, Singapore, 1991).

19. J. A. Shapiro and M. Dworkin, Bacteria as Multicellular Organisms (Oxford University Press, Oxford, 1997).
20. E. Ben-Jacob, Social behavior of bacteria: From physics to complex organization, Eur. Phys. J. B 65 (2008) 315-322.

21. J. Shong, M. R. J. Diaz and C. H. Collins, Towards synthetic microbial consortia for bioprocessing, Curr. Opin. Biotechnol., doi:10.1016/ j.copbio.2012.02.001 (212).

22. M.-O. Coppens, Nature inspired chemical engineering - learning from the fractal geometry of nature in sustainable chemical engineering, in Fractal Geometry and Applications: A Jubilee of Benoit Mandelbrot, Vol. 72 of Proceedings of Symposia Pure Mathematics, eds. M. L. Lapidus and M. van Frankenhuijsen (American Mathematical Socity, 2004), pp. 507-532.

23. M.-O. Coppens, Scaling up and down in a nature inspired way, Ind. Engng Chem. Res. 44 (2005) 5011-5019.

24. S. Kjelstrup, M.-O. Coppens, J. Pharoah and P. Pfeifer, Nature-inspired energy and material efficient design of a polymer electrolyte membrane fuel cell, Energ. Fuel 24(9) (2010) 5097-5108.

25. M.-O. Coppens, A nature-inspired approach to reactor and catalysis engineering, Curr. Opin. Chem. Eng., doi:10.1016/j.coche.2012.03.002 (2012).

26. R. E. Plotnick, R. H. Gardner, W. W. Hargrove, K. Prestegaard and M. Perlmutter, Lacunarity analysis: A general technique for the analysis of spatial patterns, Phys. Rev. E 53(5) (1996) 5461-5468.

27. C. R. Tolle, T. R. McJunkin and D. J. Gorsich, An efficient implementation of the gliding box lacunarity algorithm, Physica D 237 (2008) 306-315.

\section{APPENDIX}

\section{A.1 List of Variables}

\begin{tabular}{|c|c|l|}
\hline Variable & Unit & \multicolumn{1}{|c|}{ Description } \\
\hline$C_{n}$ & $\mathrm{~kg} / \mathrm{m}^{2}$ & Concentration of nutrients \\
\hline$m_{i}$ & $\mathrm{~kg}$ & Mass of agent $i$ \\
\hline$r_{n}$ & $\mathrm{~kg} /\left(\mathrm{m}^{2} \mathrm{~s}\right)$ & Depletion rate of nutrients per unit area \\
\hline$R\left(m_{i}\right)$ & $\mathrm{m}$ & Radius of agent $i$ \\
\hline$S_{i}$ & $\mathrm{~m}^{2}$ & Area of agent $i$ \\
\hline$t$ & $\mathrm{~s}$ & Time \\
\hline$v_{i}$ & $\mathrm{~m} / \mathrm{s}$ & Velocity of agent $i$ \\
\hline$v_{i}^{\prime}$ & Dimensionless & Dimensionless velocity of agent $i$ \\
\hline$W_{i}$ & $\mathrm{~J}$ & Amount of work done by agent $i$ \\
\hline$X_{i}$ & Dimensionless & Dimensionless mass of agent $i$ \\
\hline$Y$ & Dimensionless & Dimensionless nutrient concentration \\
\hline$\tau$ & Dimensionless & Dimensionless time \\
\hline
\end{tabular}




\section{A.2 List of Parameters}

\begin{tabular}{|c|c|l|}
\hline Parameter & Units & \multicolumn{1}{c|}{ Description } \\
\hline$a$ & $\mathrm{~m}$ & Width of the unit boxes in the nutrition grid \\
\hline$C_{\max }$ & $\mathrm{kg} / \mathrm{m}^{2}$ & $\begin{array}{l}\text { Maximum concentration of food. Since food is being depleted } \\
\text { by the agents, } C_{\text {max }} \text { is also the initial concentration of food }\end{array}$ \\
\hline$D_{C}$ & $\mathrm{~m}^{2} / \mathrm{s}$ & Diffusion coefficient of nutrients \\
\hline$F_{d}$ & $\mathrm{~N}$ & Drag force \\
\hline$\Delta H$ & $\mathrm{~J} / \mathrm{kg}$ & Enthalpy change in converting mass to metabolic energy \\
\hline$K_{M}$ & $\mathrm{~kg} / \mathrm{m}^{2}$ & Constant in Michaelis-Menten kinetics \\
\hline$m_{\min }$ & $\mathrm{kg}$ & Minimum mass of an agent \\
\hline$p$ & Dimensionless & $\begin{array}{l}\text { Stoichiometric coefficient for the conversion of nutrients } \\
\text { to agent mass }\end{array}$ \\
\hline$r_{\max }$ & $\mathrm{kg} /\left(\mathrm{m}^{2} \mathrm{~s}\right)$ & Maximum reaction rate in Michaelis-Menten kinetics \\
\hline$v_{\max }$ & $\mathrm{m} / \mathrm{s}$ & Maximum velocity of an agent \\
\hline$\mu$ & $\mathrm{kg} /(\mathrm{m} \mathrm{s})$ & Viscosity \\
\hline$\rho$ & $\mathrm{kg} / \mathrm{m}^{2}$ & Density of an agent \\
\hline
\end{tabular}

